$\begin{array}{ll}\text { Research Square } & \begin{array}{l}\text { Preprints are preliminary reports that have not undergone peer review. } \\ \text { They should not be considered conclusive, used to inform clinical practice, } \\ \text { or referenced by the media as validated information. }\end{array}\end{array}$

\title{
Altitude conditions seem to determine the evolution of COVID-19 in Brazil: a significance relationship with cases and deaths
}

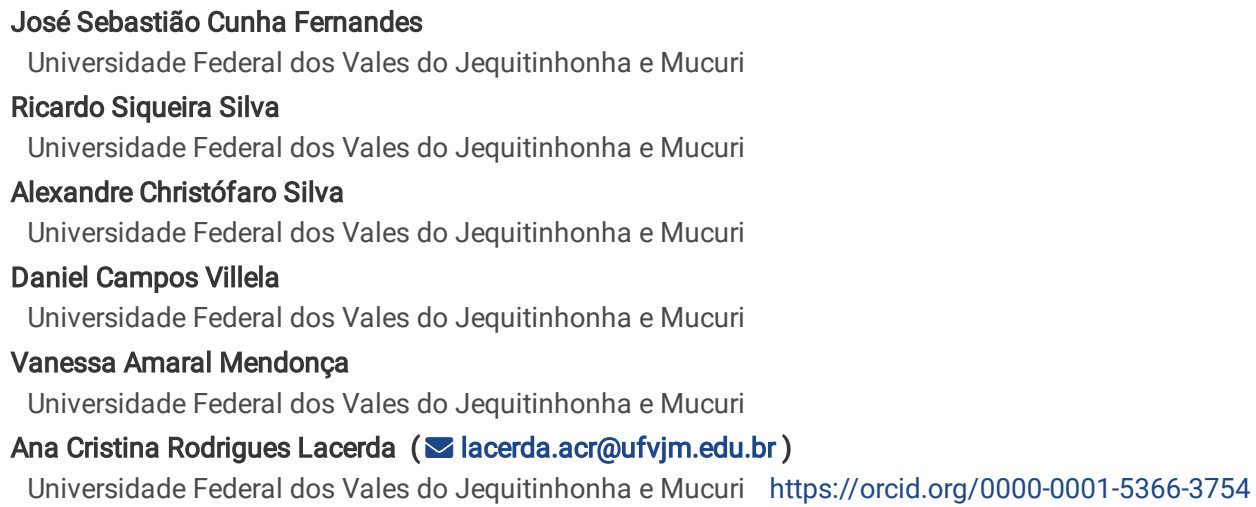

Research

Keywords: COVID-19 incidence, altitude, air humidity, environmental factors

Posted Date: September 21st, 2020

DOI: https://doi.org/10.21203/rs.3.rs-76633/v1

License: (c) (1) This work is licensed under a Creative Commons Attribution 4.0 International License. Read Full License 


\section{Abstract \\ Background}

COVID-19 is spreading rapidly in Brazil, a country of continental dimensions, but the incidence of the disease is showing to be very heterogeneous, affecting cities and regions differently. Thus, there is a gap regarding what factors would contribute to accentuate the differences in the incidence of COVID-19 among Brazilian cities. This work aimed to evaluate the effect of altitude on incidence of COVID-19 in Brazilian cities.

\section{Methods}

We analysed the relative incidence, relative death rate of COVID-19, and air relative humidity in all 154 cities in Brazil with a population above 200 thousand inhabitants, located between 5 and 1,135 $\mathrm{m}$ in altitude. Pearson's correlation analysis was performed to compare a relationship between altitude with relative incidence (RI) and relative death rate (RDR) and between air relative humidity (RH). Altitudes were classified into three classes (low class, up to 97 m; middle class, $97 \mathrm{~m}$ to $795 \mathrm{~m}$; and upper class, $795 \mathrm{~m}$ to 1,135 m) for the RI, RDR and RH variables. To compare the three classes of altitude, analysis of variance (ANOVA) and Tukey test were used to compare media $(p<0.05)$.

\section{Results}

Our epidemiological analysis found that the IR, RDR and RH were higher in cities located in low (0 to $97 \mathrm{~m}$ a.s.I) compared to medium (98 to $790 \mathrm{~m}$ a.s.I) and high (791 to $1135 \mathrm{~m}$ a.s.I) cities altitudes. Furthermore, it has been shown that there is a negative correlation between the incidence of COVID-19 with altitude and a positive correlation with air relative humidity in the cities analysed.

\section{Conclusion}

Brazilian cities at lower altitudes and greater air relative humidity have higher relative incidence and relative deaths from COVID-19. Thus, higher altitude cities may be favourable to shelter people at risk. This study may be useful for understanding the behaviour of SARS-CoV2, and start point for future studies to establish causality of environmental conditions with SARS-CoV2 contributing to the implementation of measures to prevent and control the spread of COVID19.

\section{Introduction}

In December 2019, a new coronavirus was identified - initially called 2019-nCoV and later renamed SARS-CoV-2 - in Hubei province, People's Republic of China [1]. The pathogen causes coronavirus-19 disease (COVID-19), which spread rapidly, reaching the pandemic level on March 11, 2020 [2]. The incidence of cases and deaths caused by COVID-19 in the world increased at different rates from the first cases. In Brazil, a country of continental dimensions, the spread of the disease is very heterogeneous, affecting cities and regions differently.

Efforts to minimize its spread are announced every minute in the media. Also, thousands of searches worldwide are focused on the various nuances of COVID19. However, studies that relate to the incidence of the disease to environmental factors are incipient, especially in countries like Brazil, which stands out for its great socioeconomic and environmental diversity [2]. As the behaviour of COVID-19 concerning climatic attributes is still poorly understood, investigating the influence of altitude and environmental characteristics of cities on the incidence and deaths caused by COVID-19 can generate results that contribute to the development of public policies that minimize the spread of the disease.

In Brazil, COVID-19 began to be disseminated from large cities located in the coastal zone, that have high air relative humidity, and quickly reached the interior, but the contamination speed of the population is not homogeneous [2]. Some cities have higher incidence and deaths from COVID-19 than other cities with similar numbers of inhabitants. About that, some questions were raised about the differences between the records of infection and deaths between cities. What factors would be able to contribute to accentuate these differences in the incidence of COVID-19 among Brazilian cities? Thus, the hypothesis arose that cities located at higher altitudes are less favourable for the spread of COVID-19.

Since, cities outside the coastal zone with a high incidence of COVID-19, such as Manaus - Amazonas, are located at low altitudes (< $100 \mathrm{~m}$ a.s.I). Brazil has 154 cities with a population above 200 thousand inhabitants located at altitudes ranging from 4 to $1,135 \mathrm{~m}$ a.s.l. The incidence of COVID-19 in these cities is not similar. Could altitude be a determining factor that would influence the incidence of this virus?

During the survey of epidemiological data from Brazil, in May 2020, a study was published showing the relationship between altitude and the spread of COVID-19 based on epidemiological data from China, Bolivia, and Ecuador [3]. The authors showed that inhabitants are less susceptible to develop serious adverse effects caused by COVID-19 in cities with altitudes above 3,000 m a.s.I. [3]. These authors related this low incidence to compensatory adjustments in physiological systems and environmental factors, such as higher ultraviolet radiation and thinner atmosphere.

Thus, altitude can be a determining factor in the transmission rate of COVID-19. However, due to the importance and urgency of understanding the disease and its behaviour in different locations, it is necessary to highlight the relationships of COVID-19 in other countries, especially in those with greater altitudinal and environmental diversity, such as Brazil. 
This work aims to evaluate the incidence of COVID-19 in 154 Brazilian cities with a population above 200 thousand inhabitants, located between 5 and $1,135 \mathrm{~m}$ in altitude. The results may be useful to apply public policies for the prevention and control of the spread of COVID-19 in Brazil and the world.

\section{Material And Methods}

\subsection{Cities data}

Data from all 154 Brazilian cities with a population above 200 thousand inhabitants (Fig. 1) were collected on May, June, and July, 2020. These cities are located between 5 and 1,135 meters in altitude, have 98,080,747 inhabitants (46.7\% of the Brazilian population - Brazilian Institute of Geography and Statistics - IBGE) [4]. Figure 1 shows the sampled locations, and the different altitudes in Brazil divided into three classes based on the available data DIVAGIS (http://www.diva-gis.org/Data).

\subsection{Data collect}

The altitude data for each city was carefully obtained from the average of five measurements obtained in their respective north, south, east, west, and central positions, from Google Earth at https://earth.google.com/web/@0,0,-24018.82718741a,36750128.22569847d,35y,0h,0t,0r/data=KAE (Google Earth, 2020).

The population for each city was obtained using data from IBGE, referring to July 1, 2019, at https://agenciadenoticias.ibge.gov.br/agencia-detalhe-demidia.html?view=mediaibge \&catid= $2103 \&$ id $=3098$ [4].

Data on confirmed cases and deaths, resulting from COVID-19, were obtained, on May 17, 2020, on June 01 and 16, 2020 and on July 01,2020 at https://covid.mapbiomas.org/ [5].

The data regarding air relative humidity $(\mathrm{RH})$ were obtained from the National Institute of Meteorology address http://www.inmet.gov.br/portal/index.php? $r=$ estacoes/estacoesAutomaticas [6], for the $63,58,57$ and 59 cities with a population over 200 thousand inhabitants and that have data from automatic weather stations and correspond respectively to the average of the period from March 01 to May 17, May 18 to June 01, 2020, June 02 to June 16, 2020 and June 17 to July $01,2020$.

\subsection{Statistical analysis}

The COVID-19 incidence and death data were calculated per 100,000 inhabitants and are called relative incidence (RI) and relative death rate (RDR). This objective procedure removes the effect of the number of inhabitants in the analyses.

Pearson's correlation analysis was performed to compare a relationship between altitude with RI and RDR and between RH and RI.

Define as a predetermined variable as altitudes were divided into three classes (low class, up to $97 \mathrm{~m}$, with 65 cities; middle class, $97 \mathrm{~m}$ to $795 \mathrm{~m}$, with 66 cities; and upper class, 795 m to 1,135 m, with 23 cities) for the RI, RDR variables and the same classes for the RH variable, however with 63 cities (March 01 to May 17, 2020), 58 cities (May 18 to June 01, 2020), 57 cities (June 02 to June 16, 2020), and 56 cities (June 17 to July 01, 2020).

To compare three classes of altitude (until May 17 and until June 1, 2020 for the variables RI and RDR and between March 1 and May 17 and between May 18 and June 1 for the RH variable), analysis of variance (ANOVA) and Tukey test were used to compare media $(p<0.05)$. The COVID-19 mortality and death data were transformed into a $\log (X+1)$ to stabilize the variance, and the non-transformed data were submitted to ANOVA. Both show significance. Thus, we prefer to display a statistical output of unprocessed data as analyses and figures were performed in Excel and Sigmaplot 12.5 software [7].

\section{Results}

The 154 municipalities studied correspond to $2.76 \%$ of the Brazilian municipalities (5,570 municipalities) but are home to $46.7 \%$ of the Brazilian population $(98,080,747$ inhabitants) (Table 1$)$. A total of 13 municipalities are in the central west region with $53.4 \%$ of the region's population; 12 are in the northern region with $39.59 \%$ of its population; 74 are in the south-eastern region and with $58.7 \%$ of the region's population; 28 are in the northeast region with $32.99 \%$ of the region's population, and 27 are located in the south region with $38 \%$ of the region's population. 
Table 1

Cities and regions of Brazil, altitude, population, relative incidence (RI), relative death rate (RDR), air relative humidity (RH). Data from the first case, ar

\begin{tabular}{|c|c|c|c|c|c|c|c|c|c|c|c|c|}
\hline \multirow[t]{3}{*}{ CITY } & \multirow[t]{3}{*}{ REGION } & ALTITUDE & POPULATION & RI1 & RDR1 & RH3 & $\begin{array}{l}1^{\circ} \\
\text { CASES }\end{array}$ & IA & RH4 & RI2 & RDR2 & RH5 \\
\hline & & \multirow[t]{2}{*}{$(m)$} & \multirow[t]{2}{*}{$\left(n^{0}\right)$} & $(100,000$ & $(100,000$ & \multirow[t]{2}{*}{ (\%) } & \multirow[t]{2}{*}{ (data) } & \multirow[t]{2}{*}{ (data) } & \multirow[t]{2}{*}{ (\%) } & $(100,000$ & $(100,000$ & \multirow[t]{2}{*}{ (\%) } \\
\hline & & & & hb-1) & $h b-1)$ & & & & & $h b-1)$ & $h b-1)$ & \\
\hline Cuiabá & CW & 195 & 612,547 & 44.73 & 0.33 & 63.6 & $20 /$ mar & $31 /$ mar & 62.5 & 129.79 & 1.80 & 62 \\
\hline Várzea Grande & $\mathrm{CW}$ & 195 & 284,971 & 26.67 & 1.40 & - & 23/mar & 08/may & - & 90.18 & 4.21 & - \\
\hline Rondonópolis & $\mathrm{CW}$ & 260 & 232,491 & 39.14 & 1.29 & 72.1 & $30 /$ mar & 13/apr & 69.2 & 88.61 & 3.01 & 66.7 \\
\hline Palmas & CW & 266 & 299,127 & 83.24 & 1.00 & 75.2 & $18 /$ mar & 28/apr & 69.0 & 204.26 & 2.67 & 58.4 \\
\hline Dourados & $\mathrm{CW}$ & 445 & 222,949 & 18.84 & 0.45 & 52 & $28 /$ mar & 15/may & - & 125.14 & 0.90 & 59.4 \\
\hline Campo Grande & $\mathrm{CW}$ & 590 & 895,982 & 19.42 & 0.56 & 60.4 & $14 /$ mar & 15/apr & 60.6 & 32.70 & 0.78 & 73.5 \\
\hline Rio Verde & $\mathrm{CW}$ & 740 & 235,647 & 10.61 & 0.85 & 56.4 & $12 /$ mar & 29/apr & 62.0 & 46.26 & 0.85 & - \\
\hline Goiânia & CW & 787 & $1,516,113$ & 60.75 & 1.98 & 72.5 & $12 /$ mar & $26 /$ mar & 68.2 & 114.11 & 3.50 & 62.3 \\
\hline $\begin{array}{l}\text { Aparecida de } \\
\text { Goiânia }\end{array}$ & CW & 803 & 578,179 & 26.81 & 1.21 & - & $18 /$ mar & $27 / a p r$ & - & 78.18 & 1.90 & - \\
\hline Luziânia & CW & 980 & 208,299 & 20.64 & 1.44 & 74.9 & $25 /$ mar & $14 /$ may & 70.3 & 39.37 & 1.92 & 63 \\
\hline Anápolis & $\mathrm{CW}$ & 1043 & 386,923 & 13.44 & 0.52 & - & $16 /$ mar & 12/apr & - & 40.83 & 0.52 & - \\
\hline Brasília & $\mathrm{CW}$ & 1,075 & $3,015,268$ & 112.49 & 1.79 & 78.4 & 07/mar & $24 /$ mar & 72.2 & 292.08 & 5.21 & 67.7 \\
\hline $\begin{array}{l}\text { Águas Lindas de } \\
\text { Goiás }\end{array}$ & $\mathrm{CW}$ & 1,135 & 212,440 & 13.18 & 0.47 & - & $28 /$ mar & 29/apr & - & 98.85 & 2.35 & - \\
\hline Region Mean & & 654.9 & 669,303 & & & & & & & & & \\
\hline Aracaju & $\mathrm{NE}$ & 9 & 657,013 & 309.28 & 3.04 & - & $14 /$ mar & 28/apr & - & 618.10 & 10.81 & - \\
\hline $\begin{array}{l}\text { Jaboatão dos } \\
\text { Guararapes }\end{array}$ & NE & 10 & 702,298 & 266.84 & 24.21 & - & $17 /$ mar & 07/apr & - & 454.22 & 45.28 & - \\
\hline Fortaleza & NE & 13 & $2,669,342$ & 575.83 & 43.98 & 80.2 & $16 /$ mar & $18 /$ mar & 79.9 & 902.99 & 78.07 & 79.4 \\
\hline Recife & $\mathrm{NE}$ & 17 & $1,645,727$ & 593.78 & 36.21 & 79.5 & $12 /$ mar & $28 /$ mar & 84.8 & 959.09 & 65.69 & 84.3 \\
\hline $\begin{array}{l}\text { Cabo de Santo } \\
\text { Agostinho }\end{array}$ & NE & 23 & 207,048 & 171.46 & 26.08 & - & 03/apr & $26 / a p r$ & - & 312.97 & 45.88 & - \\
\hline João Pessoa & NE & 23 & 809,015 & 209.76 & 9.27 & 78.5 & $18 /$ mar & 02/apr & 82.6 & 489.36 & 16.07 & 81.5 \\
\hline Paulista & NE & 23 & 331,774 & 354.76 & 22.00 & - & 03/apr & 09/apr & - & 568.16 & 44.91 & - \\
\hline Natal & NE & 25 & 884,122 & 134.03 & 3.96 & 82.4 & $12 /$ mar & $28 /$ mar & 87.3 & 291.36 & 9.61 & 84.2 \\
\hline Olinda & NE & 27 & 392,482 & 419.38 & 26.75 & - & $22 /$ mar & 09/apr & - & 667.04 & 43.31 & - \\
\hline Caucaia & $\mathrm{NE}$ & 31 & 361400 & 245.71 & 10.51 & - & $28 /$ mar & 13/apr & - & 499.17 & 24.90 & - \\
\hline Mossoró & NE & 32 & 297,378 & 168.81 & 7.73 & 73.7 & $21 /$ mar & 03/apr & - & 331.56 & 16.14 & - \\
\hline São Luís & NE & 32 & $1,101,884$ & 529.09 & 35.76 & 89.4 & $20 /$ mar & $30 /$ mar & 86.9 & 839.93 & 47.74 & 85.8 \\
\hline Salvador & $\mathrm{NE}$ & 36 & $2,872,347$ & 185.56 & 6.58 & 83.6 & $13 /$ mar & $21 /$ mar & 84.9 & 397.97 & 16.50 & 84.7 \\
\hline Maracanaú & NE & 41 & 227,886 & 230.82 & 27.21 & - & 29/mar & 05/apr & - & 716.15 & 50.90 & - \\
\hline Camaçari & NE & 44 & 299,132 & 31.76 & 1.34 & - & $20 /$ mar & 23/apr & - & 69.53 & 1.34 & - \\
\hline Parnamirim & NE & 44 & 261,469 & 84.14 & 2.29 & - & $20 /$ mar & 16/apr & - & 211.11 & 3.82 & - \\
\hline Itabuna & NE & 61 & 213,223 & 298.75 & 5.16 & - & 19/mar & 07/apr & - & 419.28 & 15.48 & - \\
\hline Maceió & $\mathrm{NE}$ & 65 & $1,018,948$ & 156.34 & 13.25 & 79.1 & 08/mar & 13/apr & - & 598.36 & 28.26 & - \\
\hline Sobral & $\mathrm{NE}$ & 72 & 208,935 & 261.8 & 9.09 & - & 18/mar & 22/apr & - & 1063.49 & 34.94 & - \\
\hline Teresina & NE & 115 & 864,845 & 145.69 & 4.51 & 78.9 & 19/mar & $30 /$ mar & 67.0 & 266.41 & 10.41 & 62 \\
\hline $\begin{array}{l}\text { Feira de } \\
\text { Santana }\end{array}$ & NE & 235 & 614,872 & 33.67 & 0.33 & 80.8 & 06/mar & $30 / \mathrm{mar}$ & 87.5 & 79.04 & 0.98 & 88.1 \\
\hline
\end{tabular}




\begin{tabular}{|c|c|c|c|c|c|c|c|c|c|c|c|c|}
\hline \multirow[t]{3}{*}{ CITY } & \multirow[t]{3}{*}{ REGION } & ALTITUDE & POPULATION & RI1 & RDR1 & $\mathrm{RH} 3$ & $\begin{array}{l}1^{\circ} \\
\text { CASES }\end{array}$ & IA & RH4 & $\mathrm{R} / 2$ & RDR2 & RH5 \\
\hline & & (m) & $\left(n^{0}\right)$ & $(100,000$ & $(100,000$ & $(\%)$ & (data) & (data) & $(\%)$ & $(100,000$ & $(100,000$ & $(\%)$ \\
\hline & & & & hb-1) & hb-1) & & & & & hb-1) & hb-1) & \\
\hline Arapiraca & NE & 263 & 231,747 & 56.1 & 2.59 & - & 18/apr & 27/apr & - & 180.37 & 5.18 & - \\
\hline Juazeiro & NE & 370 & 216,707 & 12 & 0.92 & - & $23 /$ mar & 14/may & - & 18.92 & 2.31 & - \\
\hline Petrolina & NE & 380 & 349,145 & 24.63 & 1.15 & 63.2 & $23 /$ mar & 06/may & 64.2 & 44.68 & 2.29 & 65.2 \\
\hline $\begin{array}{l}\text { Juazeiro do } \\
\text { Norte }\end{array}$ & NE & 408 & 274,207 & 17.87 & 2.55 & - & $20 /$ mar & 26/apr & - & 102.84 & 4.01 & - \\
\hline Campina Grande & NE & 512 & 409,731 & 70.78 & 1.22 & 80.9 & $27 /$ mar & 18/apr & 87.7 & 370.73 & 4.88 & 86.2 \\
\hline Caruaru & NE & 565 & 361,118 & 38.49 & 3.32 & 85.5 & $23 /$ mar & 18/apr & 91.4 & 104.95 & 9.14 & 91.9 \\
\hline $\begin{array}{l}\text { Vitória da } \\
\text { Conquista }\end{array}$ & NE & 970 & 341,597 & 19.61 & 1.17 & - & 01/apr & 12/apr & - & 36.89 & 1.46 & - \\
\hline Region Mean & & 158.79 & 672,335 & & & & & & & & & \\
\hline Macapá & $\mathrm{N}$ & 11 & 503,327 & 531.26 & 14.3 & 84.6 & $20 /$ mar & 03/apr & 82.3 & 1009.09 & 27.02 & 82 \\
\hline Belém & $\mathrm{N}$ & 20 & $1,492,745$ & 391.43 & 43.68 & 69.4 & 18/mar & 06/apr & 82.5 & 796.18 & 89.43 & 81.2 \\
\hline Ananindeua & $\mathrm{N}$ & 21 & 530,598 & 231.63 & 20.73 & - & $25 /$ mar & 15/apr & - & 519.98 & 45.42 & - \\
\hline Castanhal & $\mathrm{N}$ & 39 & 200,793 & 158.37 & 22.41 & 87.1 & $26 /$ mar & 19/apr & 83.8 & 357.58 & 47.31 & 81.6 \\
\hline Santarém & $\mathrm{N}$ & 44 & 304,589 & 84.7 & 5.91 & - & 01/apr & $30 / a p r$ & - & 332.58 & 30.86 & - \\
\hline Manaus & $\mathrm{N}$ & 60 & $2,182,763$ & 476.78 & 43.48 & 79.7 & 13/mar & 22/mar & 79.9 & 838.07 & 62.44 & 78.1 \\
\hline Boa Vista & $\mathrm{N}$ & 80 & 399,213 & 314.12 & 8.27 & - & $23 /$ mar & 13/apr & - & 655.04 & 24.05 & 78.6 \\
\hline Porto Velho & $\mathrm{N}$ & 86 & 529,544 & 284.96 & 9.63 & - & $21 /$ mar & 14/apr & - & 660.00 & 20.58 & - \\
\hline Marabá & $\mathrm{N}$ & 97 & 279,349 & 71.95 & 8.95 & 82.9 & 23/mar & 27/apr & 82.4 & 140.33 & 33.65 & 76.8 \\
\hline Imperatriz & $\mathrm{N}$ & 146 & 258,682 & 329.36 & 13.92 & 78.1 & 01/apr & 17/apr & 73.2 & 786.29 & 38.66 & 66.2 \\
\hline Rio Branco & $\mathrm{N}$ & 157 & 407,319 & 324.81 & 13.01 & 85.2 & 17/mar & 17/apr & - & 1007.56 & 28.48 & - \\
\hline Parauapebas & $\mathrm{N}$ & 180 & 208,273 & 90.27 & 11.04 & - & $28 /$ mar & 24/apr & - & 751.90 & 29.77 & - \\
\hline Region Mean & & 78.42 & 608,100 & & & & & & & & & \\
\hline Guarujá & SE & 9 & 320,459 & 115.46 & 4.68 & - & $31 /$ mar & 14/apr & - & 312.05 & 16.23 & - \\
\hline $\begin{array}{l}\text { Campos dos } \\
\text { Goytacazes }\end{array}$ & SE & 10 & 507,548 & 36.84 & 1.97 & 77.6 & $24 /$ mar & 27/apr & 76.4 & 146.00 & 6.11 & 79.2 \\
\hline Angra dos Reis & SE & 14 & 203,785 & 179.11 & 7.36 & 83.8 & $30 /$ mar & 28/apr & - & 437.23 & 16.68 & - \\
\hline Macaé & SE & 14 & 256,672 & 43.64 & 6.23 & 71.9 & $31 / \mathrm{mar}$ & 16/apr & 79.5 & 326.10 & 10.91 & 84.2 \\
\hline Santos & SE & 14 & 433,311 & 322.4 & 19.85 & - & $30 /$ mar & 01/apr & - & 802.66 & 34.16 & - \\
\hline Magé & SE & 15 & 245,071 & 142 & 13.87 & - & 02/apr & 23/apr & - & 267.27 & 36.32 & - \\
\hline Cabo Frio & SE & 17 & 219,863 & 60.95 & 2.73 & - & 07/apr & 23/apr & - & 171.47 & 8.19 & - \\
\hline Vitória & SE & 17 & 362,097 & 344.94 & 15.47 & 70.0 & 19/mar & 05/apr & 69.4 & 677.44 & 26.51 & 65.6 \\
\hline São Gonçalo & SE & 18 & $1,084,839$ & 58.26 & 5.99 & - & $23 /$ mar & 07/apr & - & 149.24 & 14.66 & - \\
\hline Vila Velha & SE & 18 & 493,838 & 277.82 & 9.72 & 81.6 & 19/mar & 08/apr & 81 & 490.65 & 20.05 & 82.6 \\
\hline Niterói & SE & 20 & 513,584 & 231.32 & 12.66 & 76.5 & 12/mar & $26 /$ mar & 73.7 & 596.79 & 20.25 & 79.4 \\
\hline Duque de Caxias & SE & 22 & 919,596 & 99.94 & 15.77 & 74.1 & $24 /$ mar & 07/apr & 56.5 & 163.33 & 27.19 & - \\
\hline Rio de Janeiro & SE & 23 & $6,718,903$ & 192.31 & 27.4 & 78.3 & 06/mar & 18/mar & 69.4 & 433.95 & 53.25 & 80.2 \\
\hline São Vicente & SE & 23 & 365,798 & 112.36 & 4.1 & - & $31 /$ mar & 15/apr & - & 268.73 & 21.87 & - \\
\hline Praia Grande & SE & 24 & 325,073 & 89.83 & 13.23 & - & 01/apr & 15/apr & - & 215.95 & 17.23 & - \\
\hline Itaboraí & SE & 25 & 240,592 & 152.54 & 13.3 & - & 27/mar & 04/apr & - & 470.09 & 32.42 & - \\
\hline
\end{tabular}




\begin{tabular}{|c|c|c|c|c|c|c|c|c|c|c|c|c|}
\hline \multirow[t]{3}{*}{ CITY } & \multirow[t]{3}{*}{ REGION } & ALTITUDE & POPULATION & RI1 & RDR1 & RH3 & $\begin{array}{l}1^{\circ} \\
\text { CASES }\end{array}$ & IA & RH4 & $\mathrm{R} / 2$ & RDR2 & RH5 \\
\hline & & (m) & $\left(n^{0}\right)$ & $(100,000$ & $(100,000$ & $(\%)$ & (data) & (data) & $(\%)$ & $(100,000$ & $(100,000$ & $(\%)$ \\
\hline & & & & $h b-1)$ & hb-1) & & & & & $h b-1)$ & hb-1) & \\
\hline Nova Iguaçu & SE & 27 & 821,128 & 93.29 & 11.57 & - & $28 /$ mar & 04/apr & - & 142.49 & 22.16 & - \\
\hline Belford Roxo & SE & 32 & 510,906 & 73.01 & 9.98 & - & $26 /$ mar & 11/apr & - & 139.16 & 19.77 & - \\
\hline $\begin{array}{l}\text { São João de } \\
\text { Meriti }\end{array}$ & SE & 32 & 472,406 & 99.07 & 10.16 & - & $30 /$ mar & 09/apr & - & 183.74 & 18.20 & - \\
\hline $\begin{array}{l}\text { Cachoeiro de } \\
\text { Itapemirim }\end{array}$ & SE & 62 & 208,972 & 38.28 & 1.44 & - & $20 /$ mar & 29/apr & - & 126.33 & 5.74 & - \\
\hline Cariacica & SE & 62 & 381,285 & 237.09 & 9.44 & - & $20 /$ mar & 13/apr & - & 480.22 & 24.13 & - \\
\hline Serra & SE & 65 & 517510 & 257.58 & 15.07 & - & $23 / \mathrm{mar}$ & 01/apr & - & 484.63 & 30.34 & - \\
\hline $\begin{array}{l}\text { Governador } \\
\text { Valadares }\end{array}$ & SE & 165 & 279,885 & 25.72 & 1.79 & 80.0 & $26 /$ mar & 25/apr & 80.2 & 67.17 & 2.50 & 80.6 \\
\hline Ipatinga & SE & 245 & 263410 & 13.67 & 0 & - & $12 /$ mar & 15/apr & - & 93.39 & 0.38 & - \\
\hline Volta Redonda & SE & 400 & 273,012 & 163.73 & 6.59 & - & $24 / \mathrm{mar}$ & 02/apr & - & 254.93 & 13.19 & - \\
\hline $\begin{array}{l}\text { Presidente } \\
\text { Prudente }\end{array}$ & SE & 430 & 228,743 & 39.35 & 2.19 & 58.0 & 08/apr & 24/apr & 55.2 & 60.33 & 2.62 & 69.9 \\
\hline $\begin{array}{l}\text { São José do Rio } \\
\text { Preto }\end{array}$ & SE & 518 & 460,671 & 77.93 & 2.6 & - & 18/mar & 07/apr & - & 151.95 & 4.99 & - \\
\hline Bauru & SE & 559 & 376,818 & 58.12 & 3.45 & 69.3 & $31 / \mathrm{mar}$ & 15/apr & 65.2 & 78.02 & 3.98 & 77.6 \\
\hline Piracicaba & SE & 570 & 404,142 & 55.92 & 4.21 & 64.6 & $30 /$ mar & 27/apr & 50.4 & 141.53 & 7.18 & 63.2 \\
\hline Ribeirão Preto & SE & 570 & 703,293 & 63.98 & 1.85 & - & $26 /$ mar & 04/apr & - & 134.08 & 3.41 & - \\
\hline Limeira & SE & 580 & 306,114 & 23.85 & 0.33 & - & 01/apr & 28/apr & - & 50.96 & 1.31 & - \\
\hline Americana & SE & 582 & 239,597 & 27.55 & 1.67 & - & $31 / \mathrm{mar}$ & 18/apr & - & 50.50 & 2.50 & - \\
\hline Jacareí & SE & 590 & 233,662 & 37.23 & 1.71 & - & 08/apr & 17/apr & - & 92.44 & 3.85 & - \\
\hline Rio Claro & SE & 590 & 206,424 & 12.6 & 4.36 & - & $25 /$ mar & 17/apr & - & 23.25 & 5.33 & - \\
\hline Sumaré & SE & 590 & 282,441 & 41.07 & 1.06 & - & 07/apr & 06/may & - & 68.69 & 2.48 & - \\
\hline Taubaté & SE & 595 & 314,924 & 18.73 & 1.27 & 74.1 & 19/mar & 27/apr & 70.4 & 43.82 & 1.59 & 77.9 \\
\hline Sorocaba & SE & 600 & 679,378 & 107.45 & 4.27 & 72.5 & $27 /$ mar & 25/apr & 63.5 & 135.42 & 6.48 & 76.3 \\
\hline $\begin{array}{l}\text { São José dos } \\
\text { Campos }\end{array}$ & SE & 610 & 721,944 & 62.33 & 2.77 & - & 18/mar & 04/apr & - & 124.80 & 4.85 & - \\
\hline Indaiatuba & SE & 615 & 251,627 & 22.65 & 3.97 & - & 01/apr & 17/apr & - & 58.02 & 7.95 & - \\
\hline Hortolândia & SE & 620 & 230,851 & 45.92 & 5.63 & - & 20/mar & 18/apr & - & 66.71 & 5.20 & - \\
\hline Marília & SE & 640 & 238,882 & 13.81 & 0.42 & 65.9 & 03/apr & 15/may & 57.5 & 25.95 & 0.42 & 73.7 \\
\hline Montes Claros & SE & 660 & 409,341 & 9.77 & 0.49 & 72.4 & 06/apr & 08/may & 68.2 & 16.86 & 0.49 & 62.5 \\
\hline Campinas & SE & 682 & $1,204,073$ & 81.97 & 3.07 & - & 18/mar & 07/apr & - & 151.32 & 6.48 & - \\
\hline Araraquara & SE & 694 & 236,072 & 59.73 & 1.69 & - & 02/apr & 15/apr & - & 99.12 & 1.69 & - \\
\hline Divinópolis & SE & 745 & 238230 & 53.31 & 0.42 & 80.6 & 08/mar & 01/apr & 78.7 & 79.34 & 0.84 & 76.7 \\
\hline Guarulhos & SE & 750 & $1,379,182$ & 110.79 & 11.96 & - & 17/mar & 01/apr & - & 200.77 & 20.59 & - \\
\hline Jundiaí & SE & 754 & 418,962 & 95.47 & 6.92 & - & $25 /$ mar & 16/apr & - & 245.13 & 13.61 & - \\
\hline Itaquaquecetuba & SE & 760 & 370,821 & 92.77 & 9.17 & - & $31 /$ mar & 15/apr & - & 160.45 & 19.42 & - \\
\hline Carapicuíba & SE & 770 & 400,927 & 147.16 & 9.73 & - & 14/mar & 09/apr & - & 257.90 & 18.96 & - \\
\hline Mogi das Cruzes & SE & 770 & 445,842 & 137.49 & 9.64 & - & $20 /$ mar & 10/apr & - & 223.85 & 19.07 & - \\
\hline Sete Lagoas & SE & 770 & 239,639 & 4.59 & 0.42 & 76.5 & 17/mar & 19/apr & 72.9 & 12.10 & 0.42 & 68 \\
\hline Juiz de Fora & SE & 777 & 568,873 & 72.42 & 2.81 & 73.5 & $14 /$ mar & $31 /$ mar & 76.7 & 108.46 & 5.27 & 79.5 \\
\hline
\end{tabular}




\begin{tabular}{|c|c|c|c|c|c|c|c|c|c|c|c|c|}
\hline \multirow[t]{3}{*}{ CITY } & \multirow[t]{3}{*}{ REGION } & ALTITUDE & POPULATION & RI1 & RDR1 & RH3 & $\begin{array}{l}1^{\circ} \\
\text { CASES }\end{array}$ & IA & RH4 & $\mathrm{R} / 2$ & RDR2 & RH5 \\
\hline & & $(\mathrm{m})$ & $\left(n^{\circ}\right)$ & $(100,000$ & $(100,000$ & $(\%)$ & (data) & (data) & $(\%)$ & $(100,000$ & $(100,000$ & $(\%)$ \\
\hline & & & & hb-1) & hb-1) & & & & & hb-1) & $h b-1)$ & \\
\hline Diadema & SE & 778 & 423,884 & 150.28 & 12.03 & - & $27 /$ mar & 07/apr & - & 279.09 & 19.82 & - \\
\hline Suzano & SE & 780 & 297,637 & 125.32 & 12.77 & - & $19 /$ mar & 11/apr & - & 222.08 & 16.80 & - \\
\hline Itapevi & SE & 781 & 237700 & 137.99 & 14.72 & - & $30 /$ mar & 15/apr & - & 225.07 & 26.92 & - \\
\hline Osasco & SE & 785 & 698,418 & 247.85 & 29.07 & - & $18 /$ mar & $31 / \mathrm{mar}$ & - & 389.17 & 44.53 & - \\
\hline São Paulo & SE & 785 & $12,252,023$ & 293.27 & 23.14 & 81.3 & $25 /$ fev & $11 /$ mar & 73.4 & 498.91 & 35.13 & 83.5 \\
\hline Barueri & SE & 786 & 274,182 & 265.52 & 29.91 & 76.8 & $18 /$ mar & 16/apr & 68.5 & 433.29 & 46.32 & 78.7 \\
\hline Santo André & SE & 790 & 718,773 & 180.31 & 9.46 & - & $16 /$ mar & $30 /$ mar & - & 322.49 & 18.50 & - \\
\hline Taboão da Serra & SE & 790 & 289,664 & 136.02 & 10.36 & - & $25 /$ mar & 01/apr & - & 283.78 & 22.44 & - \\
\hline Uberaba & SE & 790 & 333,783 & 29.96 & 1.50 & 75.7 & $20 /$ mar & 06/apr & 74.5 & 59.92 & 1.80 & 70.2 \\
\hline $\begin{array}{l}\text { São Bernardo do } \\
\text { Campo }\end{array}$ & SE & 795 & 838,936 & 172.36 & 14.3 & - & $16 /$ mar & $30 /$ mar & - & 313.73 & 25.27 & - \\
\hline Santa Luzia & SE & 800 & 219,134 & 9.13 & 0.46 & - & $29 /$ mar & $13 /$ may & - & 21.90 & 0.46 & - \\
\hline Mauá & SE & 805 & 472,912 & 122.01 & 8.04 & - & $16 /$ mar & 07/apr & - & 217.17 & 12.48 & - \\
\hline Embu das Artes & SE & 810 & 273,726 & 117.64 & 9.86 & - & $25 /$ mar & 02/apr & - & 202.39 & 16.81 & - \\
\hline Cotia & SE & 820 & 249210 & 139.64 & 13.64 & - & $18 /$ mar & $31 /$ mar & - & 239.96 & 20.06 & - \\
\hline Betim & SE & 830 & 439340 & 8.65 & 0.68 & - & $23 /$ mar & 06/may & - & 28.45 & 1.37 & - \\
\hline $\begin{array}{l}\text { Ribeirão das } \\
\text { Neves }\end{array}$ & SE & 870 & 334,858 & 6.87 & 0.3 & - & 02/apr & 27/apr & - & 24.19 & 0.60 & - \\
\hline São Carlos & SE & 870 & 251,983 & 22.62 & 1.19 & 68.0 & 06/apr & 09/may & 64.9 & 48.81 & 1.98 & 72.7 \\
\hline Petrópolis & SE & 889 & 306,191 & 69.56 & 8.82 & - & $21 /$ mar & 14/apr & - & 136.84 & 13.72 & - \\
\hline Uberlândia & SE & 892 & 691,305 & 56.27 & 1.59 & 64.2 & $17 /$ mar & 02/apr & 63.3 & 130.04 & 2.60 & 56.1 \\
\hline Belo Horizonte & SE & 904 & $2,512,070$ & 46.18 & 1.23 & 72.9 & $16 /$ mar & $23 /$ mar & 75.2 & 74.92 & 1.95 & 71 \\
\hline Contagem & SE & 953 & 663,855 & 16.87 & 0.45 & - & $23 /$ mar & 17/apr & - & 39.32 & 1.51 & - \\
\hline Franca & SE & 962 & 353,187 & 10.19 & 0.57 & 66.4 & 02/apr & 28/apr & 62.1 & 23.22 & 0.57 & - \\
\hline Region Mean & & 500.4 & 700,862 & & & & & & & & & \\
\hline Rio Grande & $\mathrm{S}$ & 5 & 211,005 & 4.74 & 0.47 & - & $23 /$ mar & 02/may & - & 9.00 & 0.47 & - \\
\hline Itajaí & $\mathrm{S}$ & 6 & 219,536 & 80.17 & 1.37 & 80.0 & $21 /$ mar & $27 /$ mar & 75.6 & 209.99 & 4.56 & 86.3 \\
\hline Pelotas & $\mathrm{S}$ & 12 & 342,405 & 10.22 & 0 & - & $25 /$ mar & 14/apr & - & 25.12 & 0.00 & - \\
\hline Canoas & $\mathrm{S}$ & 13 & 346,616 & 13.85 & 0.87 & - & $21 /$ mar & 17/apr & - & 29.72 & 1.73 & - \\
\hline Florianópolis & $\mathrm{S}$ & 22 & 500,973 & 94.42 & 1.2 & 70.6 & $12 /$ mar & $23 /$ mar & 71.1 & 137.13 & 1.40 & 77.7 \\
\hline Gravataí & $\mathrm{S}$ & 32 & 281,519 & 10.66 & 0.36 & - & $25 /$ mar & 02/apr & - & 22.38 & 1.42 & - \\
\hline Joinville & $\mathrm{S}$ & 32 & 590,466 & 52.16 & 1.86 & - & $13 /$ mar & $30 /$ mar & - & 71.64 & 3.90 & - \\
\hline Novo Hamburgo & $\mathrm{S}$ & 37 & 246,748 & 29.99 & 0.81 & - & $29 /$ mar & 03/apr & - & 60.39 & 1.62 & - \\
\hline São Leopoldo & $\mathrm{S}$ & 42 & 236,835 & 48.13 & 0.42 & - & $21 /$ mar & 09/apr & - & 97.54 & 0.84 & - \\
\hline São José & $\mathrm{S}$ & 44 & 246,586 & 36.09 & 0.41 & - & 19/mar & 09/apr & - & 50.29 & 0.81 & - \\
\hline Blumenau & $\mathrm{S}$ & 47 & 357,199 & 117.02 & 0.84 & - & $22 /$ mar & 06/apr & - & 184.49 & 1.12 & - \\
\hline Criciúma & $\mathrm{S}$ & 52 & 215,186 & 130.58 & 2.79 & - & $20 /$ mar & 09/apr & - & 178.45 & 4.18 & - \\
\hline Alvorada & $\mathrm{S}$ & 57 & 210,305 & 10.94 & 3.8 & - & 19/mar & 17/apr & - & 31.86 & 1.43 & - \\
\hline Porto Alegre & $\mathrm{S}$ & 58 & $1,483,771$ & 40.57 & 1.42 & 71.7 & $11 /$ mar & $20 /$ mar & 76.3 & 50.14 & 2.49 & 83.0 \\
\hline Viamão & $S$ & 92 & 255,224 & 12.93 & 0.39 & - & $23 /$ mar & 13/apr & - & 18.42 & 1.57 & - \\
\hline
\end{tabular}




\begin{tabular}{|c|c|c|c|c|c|c|c|c|c|c|c|c|}
\hline \multirow[t]{3}{*}{ CITY } & \multirow[t]{3}{*}{ REGION } & ALTITUDE & POPULATION & Rl1 & RDR1 & RH3 & $\begin{array}{l}1^{\circ} \\
\text { CASES }\end{array}$ & IA & RH4 & $\mathrm{R} / 2$ & RDR2 & RH5 \\
\hline & & \multirow{2}{*}{ (m) } & \multirow{2}{*}{$\left(n^{0}\right)$} & $(100,000$ & $(100,000$ & \multirow{2}{*}{$(\%)$} & \multirow{2}{*}{ (data) } & \multirow{2}{*}{ (data) } & \multirow{2}{*}{$(\%)$} & $(100,000$ & $(100,000$ & \multirow[t]{2}{*}{ (\%) } \\
\hline & & & & hb-1) & hb-1) & & & & & hb-1) & hb-1) & \\
\hline Santa Maria & s & 116 & 282,123 & 15.6 & 0.71 & 68.9 & $22 /$ mar & 08/apr & 78.9 & 65.57 & 1.06 & 86.2 \\
\hline Foz do Iguaçu & $\mathrm{s}$ & 202 & 258,532 & 29.01 & 0.77 & 63.3 & $18 /$ mar & 29/mar & 66.3 & 48.74 & 0.77 & 71.0 \\
\hline Maringá & s & 518 & 423,666 & 21.72 & 1.42 & 56.3 & $18 /$ mar & 21/apr & 57.7 & 38.71 & 1.42 & 74.1 \\
\hline Londrina & S & 554 & 569,733 & 23.52 & 2.81 & - & $17 /$ mar & 01/apr & - & 74.25 & 4.21 & - \\
\hline Passo Fundo & $s$ & 661 & 203,275 & 152.5 & 10.82 & 66.2 & $26 /$ mar & 21/apr & 75.9 & 364.53 & 15.25 & 82.7 \\
\hline Chapecó & $\mathrm{s}$ & 671 & 220,367 & 229.62 & 0 & - & $20 /$ mar & 26/apr & - & 419.75 & 1.82 & - \\
\hline Cascavel & S & 743 & 328,454 & 42.02 & 1.52 & - & $23 / \mathrm{mar}$ & 03/apr & - & 140.05 & 2.13 & - \\
\hline Caxias do Sul & s & 758 & 510,906 & 14.68 & 0.39 & - & $12 / \mathrm{mar}$ & $30 / \mathrm{mar}$ & - & 32.69 & 0.78 & - \\
\hline $\begin{array}{l}\text { São José dos } \\
\text { Pinhais }\end{array}$ & s & 910 & 323340 & 14.85 & 0.93 & - & $29 / \mathrm{mar}$ & $12 /$ may & - & 32.16 & 1.24 & - \\
\hline Ponta Grossa & S & 930 & 351,736 & 9.1 & 0 & - & $21 / \mathrm{mar}$ & 23/apr & - & 20.47 & 0.00 & - \\
\hline Curitiba & s & 940 & $1,933,105$ & 33.06 & 1.71 & 71.6 & $12 /$ mar & $19 /$ mar & 59.8 & 50.90 & 2.43 & 69.1 \\
\hline Colombo & S & 998 & 243,726 & 11.9 & 0 & - & $22 / \mathrm{mar}$ & 04/may & - & 20.51 & 0.00 & - \\
\hline Region Mean & & 316.7 & 421,975 & & & & & & & & & \\
\hline Total & & & $98,080,747$ & & & & & & & & & \\
\hline
\end{tabular}

IA: Incidence acceleration; RH: air relative humidity; RI: relative incidence; RDR: relative death rate; CW: central-west; NE: northeast; N: north; SE: southeast; S: $\mathrm{s}$ between March 01 and May, 17, 2020. 4: between May 18 and June, 01, 2020.5: 4: between June 02 and June, 16, 2020. 6: until June, 16, 2020; 7: between Ji

On May 17, 2020, these municipalities concentrated $71 \%$ of the cases $(171,287)$ and $78 \%(12,610)$ of deaths due to COVID-19 in Brazil. On June, 01,2020 , these municipalities concentrated $63 \%$ of the cases $(330,271)$ and $76 \%(22,567)$ of deaths due to COVID-19 in Brazil. On June, 16,2020 , these municipalities concentrated $59 \%$ of the cases $(544,408)$ and $72 \%(32,829)$ of deaths due to COVID-19 in Brazil. On July, 01, 2020, these municipalities concentrated 56\% of the cases $(815,016)$ and $66 \%(42,949)$ of deaths due to COVID-19 in Brazil. The decrease in RDR in cities with a population over 200 thousand inhabitants, between May, 17 and July, 01, indicates that the COVID-19 virus is spreading to smaller cities.

The altitude varies between 5 and 1,135 $\mathrm{m}$ a.s.I., and the cities located at higher altitudes are in the central-west region, followed by the southeast and south regions. The northern region has the lowest altitude gradient, and all cities are below $180 \mathrm{~m}$ a.s.l. (Table 1).

On May 17, 2020, the RI varied between 4.74 (Rio Grande) and 593.78 (Recife) (Table 1) and was higher in the north, followed by the northeast, southeast, central-west and, south (Table 2). On June 01, 2020, the RI varied between 12.10 (Sete Lagoas) and 1,063.49 (Sobral) (Table 1) and was higher in the north, followed by the northeast, southeast, central-west and, south (Table 2). On June 16, 2020, the RI changed between 18.78 (Sete Lagoas) and 2,998.95 (Parauapebas) (Table 1) and was also higher in the north, followed by the northeast, southeast, central-west, and south (Table 2). On July 01, 2020, the RI changed between 64.98 (Montes Claros) and 4,758.18 (Parauapebas) (Table 1) and was higher in the north, followed by the northeast, central-west, southeast, and south (Table 2).

Table 2

Mean relative incidence $(\mathrm{RI})$, mean relative death rate $(\mathrm{RDR})$, mean air relative humidity $(\mathrm{RH})$ by geographic region and in Brazil.

\begin{tabular}{|c|c|c|c|c|c|c|c|c|c|c|c|c|}
\hline \multirow[b]{2}{*}{ Region } & \multicolumn{4}{|l|}{ RI } & \multicolumn{3}{|l|}{ RDR } & \multicolumn{3}{|c|}{$\mathrm{RH}(\%)$} & \multirow[b]{2}{*}{ June/16 } & \multirow[b]{2}{*}{ July/0 } \\
\hline & May /17 & June/01 & June/16 & July/01 & May /17 & June/01 & June/16 & July/01 & May /17 & June/01 & & \\
\hline NO & 357.07 & 739.67 & 1163.09 & 1619.31 & 28.33 & 52.64 & 71.29 & 81.58 & 81.0 & 80.7 & 77.8 & 75.0 \\
\hline NE & 284.35 & 529.16 & 837.25 & 1163.52 & 17.34 & 32.06 & 48.87 & 62.36 & 79.7 & 82.2 & 81.2 & 78.0 \\
\hline SE & 156.84 & 297.06 & 483.79 & 719.44 & 13.5 & 22.45 & 35.59 & 44.87 & 73.3 & 69.1 & 74.3 & 69.1 \\
\hline SU & 42.45 & 75.90 & 136.16 & 273.63 & 1.40 & 2.18 & 3.57 & 6.49 & 68.6 & 70.2 & 78.8 & 73.4 \\
\hline $\mathrm{CO}$ & 60.46 & 160.78 & 407.94 & 884.84 & 1.34 & 3.23 & 6.86 & 14.31 & 67.3 & 66.8 & 64.1 & 59.0 \\
\hline Brazil Mean & 174.6 & 336.73 & 555.06 & 905.91 & 12.90 & 23.01 & 33.47 & 45.06 & 74.0 & 73.8 & 75.2 & 70.9 \\
\hline
\end{tabular}

In Brazil, between May 17 and June 01, between June 02 and June 16, and between June 17 and July, 01, 2020, the RI increased 92.3\% 64.8\%, 63,20\%, respectively. The largest increase in RI was observed in the central-west region and the smallest in the northeast region (Table 2), and the rate of RI growth 
decelerated in all regions, except in the southern region (Fig. 2).

On May 17, 2020, the RDR varied between 0 (Chapecó, Colombo, Ipatinga, Pelotas and, Ponta Grossa) and 43.98 (Fortaleza) (Table 1) and was higher in the north, followed by the northeast, southeast, south and central-west (Table 2). On June 01, 2020, the RDR varied between 0 (Colombo, Pelotas and, Ponta Grossa) and 78.07 (Fortaleza) (Table 1) and was higher in the north, followed by the northeast, southeast, central-west and south (Table 2). On June 16, 2020, the RDR changed between 0 (Pelotas) and 119.11 (Belém) (Table 1). On July 01, 2020, the RDR changed between 0.28 (Ponta Grossa) and 128.62 (Belém) (Table 1) and was higher in the north, followed by the northeast, southeast, central-west and south (Table 2).

Between May 17 and June 01, between June 02 and June 16, and between June 17 and July 01, the RDR increased respectively 78.4\%, 45.5\% and 34,6\% in Brazil. The largest increase in RDR was observed in the central-west region and the smallest in the north region (Table 2), and the rate of RDR growth decelerated in all regions, except in the southern region (Fig. 2).

Between March 01 and May 17, 2020, the average air relative humidity (RH) data of 63 cities were analysed, which varied between 52 and $89.4 \%$ (Table 1) with an $\mathrm{RH}$ average of $74 \%$ (Table 2). Between May 17 and June 01, 2020, the average air relative humidity (RH) data of 58 cities were analysed, which varied between 50.4 and $91.4 \%$ (Table 1) with an RH average of 73.8\% (Table 2). Between June 02 and June 16, 2020, the average air relative humidity (RH) of 57 cities varied between 56.1 and $91.9 \%$ (Table 1) with an RH of 75.2\% (Table 2). Between June 17 and July 01, 2020, the average air relative humidity (RH) of 58 cities varied between 50.3 and $92.5 \%$ (Table 1) with an $\mathrm{RH}$ of 70,9\% (Table 2). The cities in the central-west region had the lowest average $\mathrm{RH}$ values. Cities in the northern and northeast region had the highest $\mathrm{RH}$ values (Table 1).

The first confirmed case in Brazil occurred on February 26 of 2020 in the city of São Paulo, and until April 8 of 2020, all cities in Brazil with a population of up to 200 thousand inhabitants confirmed at least one case of COVID-19 (Table 1). There were no significant differences between cities concerning the period of accelerated dissemination. Would, then, the altitude and the air relative humidity of the air have contributed to accelerate the spread of the virus in Brazil?

The correlation between altitude and RI calculated from data collected in 154 cities until May 17, 2020, was negative and significant, according to Pearson's correlation $(r=-0.38, n=154, p<0.01)$. The correlation between altitude and RI calculated from data collected in 154 cities until June 01, 2020, was higher $(r=$ $-0.44, n=154, p<0.01)$. The correlation between altitude and RI calculated from data collected in 154 cities until June 16, 2020, was also negative and significant $(r=-0.41, n=154, p<0.01)$. The correlation between altitude and RI calculated from data collected in 154 cities until July 01,2020 , was also negative and significant $(r=-0.36, n=154, p<0.01)$. The same occurred between altitude and RDR until May $17,2020(r=-0.29, n=154, p<0.01)$, until June 01, $2020(r=-0.37, n=154, p<0.01)$, until June 16, $2020(r=-0.41, n=154, p<0.01)$ and until July 01, $2020(r=-0.43, n=154, p<0.01)$.

The correlation between $\mathrm{RH}$ and RI calculated from data collected in the period from March 01 to May 17, 2020, in 63 cities $(r=0.53, n=63, p<0.01)$, in the period from May 18 to June 01,2020 , in 58 cities ( $r=0.47, n=58, p<0.01)$, in the period from June 02 to June 16,2020 , in 57 cities $(r=0.28, n=57, p<0.01)$, and in the period from June 17 to July 01,2020 , in 56 cities, $(r=0.30, n=56, p<0.05)$ was positive and significant.

The correlation between RH and RDR calculated from data collected in the period from March 01 to May 17, 2020, in 63 cities ( $r=0.46, n=63, p<0.01$ ), in the period from May 18 to June 01, $2020(r=0.37, n=58, p<0.01)$, in the period from June 02 to June 16,2020 , in 57 cities $(r=0.32, n=57, p<0.01)$, and in the period from June 17 to July 01,2020 , in 56 cities $(r=0.31, n=56, p<0.05)$, was positive and significant.

The cities were grouped into three classes of altitude: low (0 to $97 \mathrm{~m}$ a.s.I), medium (98 to $790 \mathrm{~m}$ a.s.l.) and high (791 to $1.135 \mathrm{~m}$ a.s.I.) (Fig. 2 ). For RI, RDR and $\mathrm{RH}$, the analysis of variance (hierarchical classification) for data collected until May 17, 2020 (RI: $F=18.80 ; p<0.001 ; R D R: F=12.46 ; p<0.001 ; R H: F=$ 7.35; $p<0.001$ ), for data collected until June 01, 2020 (RI: $F=19.40 ; p<0.001$; RDR: $F=18.16 ; p<0.001 ; R H: F=8.00 ; p<0.001$ ), for data collected until June 16, 2020 (RI: $F=13.40 ; p<0.001 ; R D R: F=20.40 ; p<0.001 ; R H: F=10.80 ; p<0.001$ ), and for data collected until July 01, 2020 (RI: $F=9,34 ; p<0.001 ; R D R: F=$ 21,$31 ; p<0.001 ; R H: F=8,34 ; p<0.001$ ) was highly significant. The means of RI, RDR, and RH were compared using the Tukey test, which showed that the incidence is higher at low altitudes in the four analysed periods (Fig. 3).

The first confirmed case in Brazil occurred on February 26 of 2020 in the city of São Paulo, and until April 8 of 2020, all cities in Brazil with a population of up to 200 thousand inhabitants confirmed at least one case of COVID-19 (Table 1). There were no significant differences between cities concerning the period of accelerated dissemination. The $r$ values of Pearson's correlation between altitude and RI and RDR and the F values of ANOVA for all dependent variables increased from the first to the second analysed period. Would, then, the altitude and the air relative humidity have contributed to accelerate the spread of the virus in Brazil?

\section{Discussion}

Our epidemiological analysis of the COVID-19 pandemic in Brazil indicates a direct association between the incidence of COVID-19 with altitude and RH in Brazilian cities with a population above 200 thousand inhabitants. The highest RI and RDR in cities with lower altitudes may be related to environmental factors, which influence the spread of the virus and the physiology of human beings.

Climatic factors can be determinant for transmission by some viruses [8]. Studies have shown that transmission of the influenza virus is more favourable in tropical and subtropical countries than in countries with temperate climates [8]. However, it is necessary to consider comprehensive biophysical assessments of altitude, humidity, UV radiation in the maintenance and transmission of SARS-CoV-2. $[9,10]$. Recently studies have been published about temperature and UV index, and that heating, and UV irradiation can eliminate the viral infectivity $[9,10]$. These findings could help to understand the relationship of COVID-19 cases in different cities Brazil due to altitude. 
In contrast to what other studies report regarding the greater spread of the influenza virus in environments with lower air relative humidity [11], our data show that COVID-19 RI is higher in cities where RH is highest. These results are in line with those obtained by C Arias-Reyes, N Zubieta-DeUrioste, L Poma-Machicao, F Aliaga-Raudan, F Carvajal-Rodriguez, M Dutschmann, E Schneider-Gasser, G Zubieta-Calleja and J Soliz [3], which lists air dryness as one of the factors that control the spread of the virus at high altitudes. In working with climatic data from 5 large Brazilian cities (Manaus, Fortaleza, Brasília, Rio de Janeiro, and São Paulo), [12] showed that moderate relative air humidity (averages between 77.7 and $81.6 \%$ ) favor the spread of this disease. In the present study, 5 cities with the highest $\mathrm{RI}$, in the four periods analyzed, had an average $\mathrm{RH}$ between 79.3 and $84.8 \%$, values similar to those found by $\mathrm{A}$ Auler, $\mathrm{F}$ Cássaro, $\mathrm{V}$ da Silva and $\mathrm{L}$ Pires [12].

Another point that must be considered is that the higher altitude in itself favors a higher incidence of ultraviolet (UV) radiation, especially in the UVA and UVB spectra, which can produce a bactericidal effect due to changes in the molecular chains of DNA and RNA. Thus, as a hypothesis, UV radiation would shorten the virus half-life, thereby reducing the virus's ability to survive in Brazilian cities located at higher altitudes and, consequently, the survival of the COVID-19 virus. Besides, considering that vitamin D production is dependent on exposure to UV radiation and that vitamin D levels positively modulate the immune system [13], the hypothesis of higher immune defense against SARS-CoV- 2 is plausible in cities with higher altitude. Future studies should investigate this hypothesis.

The lower air density and greater distance between molecules in Brazilian cities located at higher altitudes could also reduce the inoculation of airborne viruses compared to sea level. C Arias-Reyes, N Zubieta-DeUrioste, L Poma-Machicao, F Aliaga-Raudan, F Carvajal-Rodriguez, M Dutschmann, E SchneiderGasser, G Zubieta-Calleja and J Soliz [3] suggested that inhabitants of cities with altitudes above 3,000 m a.s.l. are less susceptible to developing effects caused by COVID-19 due to ultraviolet radiation and thinner air.

Physiological factors can influence the pathogenicity of SARS-CoV-2 at high altitudes. The barometric reading varies with changing weather conditions and becomes lower as the altitude increases. Thus, the volumes of inspired air, which require humidification, are much higher than at sea level, and the air density is lower at high altitudes [14]. As a result, compensatory adjustments to facilitate the release of oxygen to cells occur in individuals living at higher altitudes, such as the increase in the levels of 2,3-diphosphoglycerate (2,3-DPG), a chemical compound found inside the red blood cell, whose function is to reduce hemoglobin's affinity for oxygen in order to facilitate its release into tissues [15]. Given the above, a probable hypothesis for less severity in individuals infected with COVID-19 living in cities with higher altitude could be due to adaptations in these compensatory adjustments to increase the bioavailability of peripheral oxygen. This adaptation is proven in individuals who live at altitudes above 3,000 m a.s.l. In our study, RDR was higher at low altitudes. In this way, such adaptations, even to a lesser extent, could contribute to minimizing the severity of infection in cities located at higher altitudes.

The casual movement of dissolved oxygen molecules establishes the PO2 of plasma and tissue fluids. The pressure of oxygen in the solution helps to regulate breathing, particularly at higher altitudes, when the ambient PO2 decreases considerably; it also determines the uptake of oxygen by haemoglobin in the lungs and the subsequent release into the tissues. However, haemoglobin saturation with oxygen changes very little until the oxygen pressure has decreased to about $60 \mathrm{mmHg}$. Even when alveolar PO2 drops to $75 \mathrm{mmHg}$, as it does at high altitudes, haemoglobin saturation decreases by only approximately $6 \%$. At $60 \mathrm{mmHg}$ alveolar P02, haemoglobin is still $90 \%$ saturated with oxygen. Below that pressure, the volume of oxygen combined with haemoglobin decreases more quickly.

As exposed, the $\mathrm{S}$ shape of the oxyhaemoglobin dissociation curve indicates that there is only a small change in the percentage saturation of haemoglobin with oxygen up to an altitude of approximately $3,048 \mathrm{~m}$. At 1,981 $\mathrm{m}$, for example, the alveolar PO2 falls from its value at sea level from $100 \mathrm{mmHg}$ to $78 \mathrm{mmHg}$. However, haemoglobin remains $90 \%$ saturated with oxygen. Given the above, considering that the Brazilian cities studied at a higher altitude are around $1100 \mathrm{~m}$, probably the hypothesis of compensatory adjustments related to ACE2 in pulmonary epithelial cells, i.e., a protective factor for virus penetration and evolution of severe pulmonary edema, should be studied with caution in residents of Brazilian cities with higher altitudes. Furthermore, this population is also not exposed to conditions of chronic hypoxia.

Also, regarding the possible physiological factors, the receptor-binding domain (RBD) in the SARS-CoV-2 protein was recently identified and that the RBD protein-bound firmly to the receptors of the human angiotensin-converting enzyme 2 (ACE2) [16]. Human ACE2 is part of the renin-angiotensin system (RAS), an essential hormonal system for controlling blood pressure and fluid and electrolyte balance. In the classical view, RAS peptides are generated from a single precursor protein called angiotensinogen (ATG). After being cleaved by the protease renin, this protein forms the inactive decapeptide angiotensin I, which is hydrolysed by the angiotensin-converting enzyme (ACE) and forms the octapeptide angiotensin II (Ang II), the principal peptide in the system. ACE2 cleaves a single residue of angiotensin I (Ang I) that generates the Ang- (1-9) peptide and degrades Ang II to the Ang- (1-7) vasodilator. Current data obtained during the pandemic suggest that the use of ACE inhibitors and angiotensin type I receptor blockers increase the expression of ACE2. Consequently, increased expression of ACE2 would facilitate infection by COVID-19 [17, 18]. Studies show that RAS elements are modulated at high altitudes [19-21], the expression of the ACE2 enzyme can be down-regulated due to the high altitude favouring a lower incidence of COVID-19 infection.

The results presented in this work can be useful for the implementation of public policies for prevention, control of the dissemination of COVID-19 in Brazil and the world. Also, it can contribute to future studies, including other zoonotic viruses that cause respiratory diseases, as well as allowing the recommendation of changing the environment for people at risk in COVID-19.

Our findings identified that virulence by SARS-CoV-2 is higher in Brazilian cities with a population above 200 thousand inhabitants, located at the lowest altitudes, and where the RH is highest (Fig. 3). These findings are in line with the physiological compensatory adjustments of the inhabitants of cities located at higher altitudes, as well as with the common characteristics. Thus, our study starts point for future studies to establish causality of environmental conditions with SARS-CoV2, contributing to the implementation of measures to prevent and control the spread of COVID-19. It is, however, important to note that the information presented here clearly lacks any physiological evidences, which may merit further investigation. As prospective, longitudinal studies are needed to confirm whether these associations remain over time. 


\section{Declarations}

\section{ETHICS APPROVAL AND CONSENT TO PARTICIPATE}

Not applicable.

\section{CONSENT FOR PUBLICATION}

The researchers of this study confirm that they have given due consideration to protect the intellectual property associated with this work and that there are no impediments to publication, including the timing of publication, with respect to intellectual property. In so doing we confirm that we have followed the regulations of our institutions concerning intellectual property.

\section{AVAILABILITY OF SUPPORTING DATA}

The datasets used and/or analysed during the current study are available from the corresponding author on reasonable request.

\section{COMPETING INTERESTS}

The authors declare that they have no known competing financial interests or personal relationships that could have appeared to influence the work reported in this paper.

The authors declare no financial interests/personal relationships which may be considered as potential competing interests.

\section{FUNDING}

There are currently no Funding Sources in the list

\section{AUTHORS' CONTRIBUTIONS}

José Sebastião Cunha Fernandes: Formal analysis, Data Curation, Methodology

Ricardo Siqueira da Silva: Formal analysis, Data Curation, Methodology, Writing

- Review \& Editing - Original Draft,

Alexandre Christófaro Silva: Formal analysis, Data Curation, Methodology, Writing - Review \& Editing

Daniel Campos Villela: Writing - Review \& Editing

Vanessa Amaral Mendonça: Writing - Review \& Editing

Ana Cristina Rodrigues Lacerda: Conceptualization, Data Curation, Writing

- Review \& Editing - Original Draft

\section{ACKNOWLEDGMENTS}

We thank the Universidade Federal dos Vales do Jequitinhonha e Mucuri for institutional support. The CNPq, CAPES- Finance Code 001, and FAPEMIG for support and scholarships.

\section{References}

1. Huang X, Wei F, Hu L, Wen L, Chen K. Epidemiology and clinical characteristics of COVID-19. Arch Iran Med. 2020;23(4):268-71.

2. World Health Organization https://www.who.int/dg/speeches/detail/who-director-general-s-opening-remarks-at-the-media-briefing-on-covid-19--11-march2020.

3. Arias-Reyes C, Zubieta-DeUrioste N, Poma-Machicao L, Aliaga-Raudan F, Carvajal-Rodriguez F, Dutschmann M, Schneider-Gasser E, Zubieta-Calleja G, Soliz J: Does the pathogenesis of SAR-CoV-2 virus decrease at high-altitude? Respiratory Physiology \& Neurobiology 2020:103443.

4. Instituto Brasileiro de Geografia e Estatística [https://agenciadenoticias.ibge.gov.br/agencia-detalhe-de-midia.html? view=mediaibge\&catid=2103\&id=3098 ].

5. Sistema. de Estimativas de Emissões de Gases de Efeito Estufa do Observatório do Clima. https://covid.mapbiomas.org/.

6. Instituto nacional de meteorologia [http://www.inmet.gov.br/portal/index.php?r=estacoes/estacoesAutomaticas ].

7. SigmaPlot. SigmaPlot version 12.5. In.: Systat Software, Inc San Jose, California; 2012

8. Lowen AC, Steel J. Roles of humidity and temperature in shaping influenza seasonality. Journal of virology. 2014;88(14):7692-5.

9. Gunthe SS, Swain B, Patra SS, Amte A. On the global trends and spread of the COVID-19 outbreak: preliminary assessment of the potential relation between location-specific temperature and UV index. Journal of Public Health 2020:1-10. 
10. Duan S, Zhao X, Wen R, Huang J-j, Pi G, Zhang S, Han J, Bi S, Ruan L. Dong X-p: Stability of SARS coronavirus in human specimens and environment and its sensitivity to heating and UV irradiation. Biomed Environ Sci: BES. 2003;16(3):246-55.

11. Casanova LM, Jeon S, Rutala WA, Weber DJ, Sobsey MD. Effects of air temperature and relative humidity on coronavirus survival on surfaces. Appl Environ Microbiol. 2010;76(9):2712-7.

12. Auler A, Cássaro F, da Silva V, Pires L. Evidence that high temperatures and intermediate relative humidity might favor the spread of COVID-19 in tropical climate: A case study for the most affected Brazilian cities. Science of The Total Environment 2020:139090.

13. Hart PH, Gorman S. Exposure to UV wavelengths in sunlight suppresses immunity. To what extent is UV-induced vitamin D3 the mediator responsible? The Clinical Biochemist Reviews. 2013;34(1):3.

14. Hodkinson P. Acute exposure to altitude. BMJ Military Health. 2011;157(1):85-91.

15. Mulquiney PJ, Kuchel PW. Model of 2, 3-bisphosphoglycerate metabolism in the human erythrocyte based on detailed enzyme kinetic equations: computer simulation and metabolic control analysis. Biochem J. 1999;342(3):597-604.

16. Tai W, He L, Zhang X, Pu J, Voronin D, Jiang S, Zhou Y, Du L. Characterization of the receptor-binding domain (RBD) of 2019 novel coronavirus: implication for development of RBD protein as a viral attachment inhibitor and vaccine. Cellular \& molecular immunology 2020:1-8.

17. Fang L, Karakiulakis G, Roth M: Are patients with hypertension and diabetes mellitus at increased risk for COVID-19 infection? The Lancet Respiratory Medicine 2020, 8(4):e21.

18. Patel AB, Verma A: COVID-19 and angiotensin-converting enzyme inhibitors and angiotensin receptor blockers: what is the evidence? Jama 2020.

19. Hogan R 3rd, Kotchen TA, Boyd 3rd A, Hartley LH. Effect of altitude on renin-aldosterone system and metabolism of water and electrolytes. J Appl Physiol. 1973;35(3):385-90.

20. Colice GL, Ramirez G. Effect of hypoxemia on the renin-angiotensin-aldosterone system in humans. J Appl Physiol. 1985;58(3):724-30.

21. Epstein M, Saruta T. Effects of simulated high altitude on renin-aldosterone and Na homeostasis in normal man. J Appl Physiol. 1972;33(2):204-10.

\section{Figures}

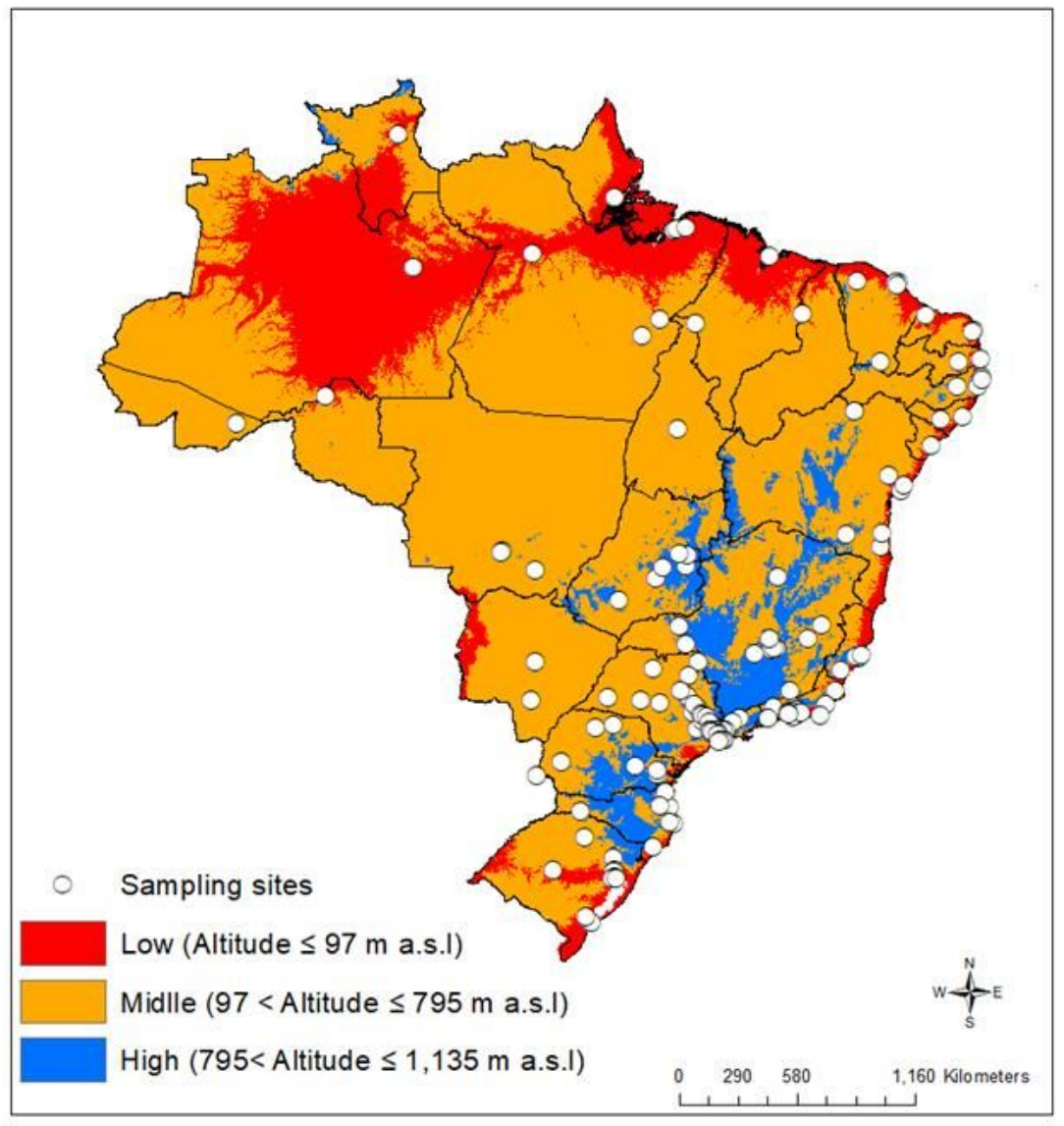

Figure 1

Distribution of 154 Brazilian cities with a population above 200 thousand inhabitants selected to analyse the relative incidence and relative deaths per 100.000 inhabitants of Coronavirus Disease-19 (COVID-19) cases according to three classes of altitudes. low (red), medium (orange) and high (blue). 
(A)

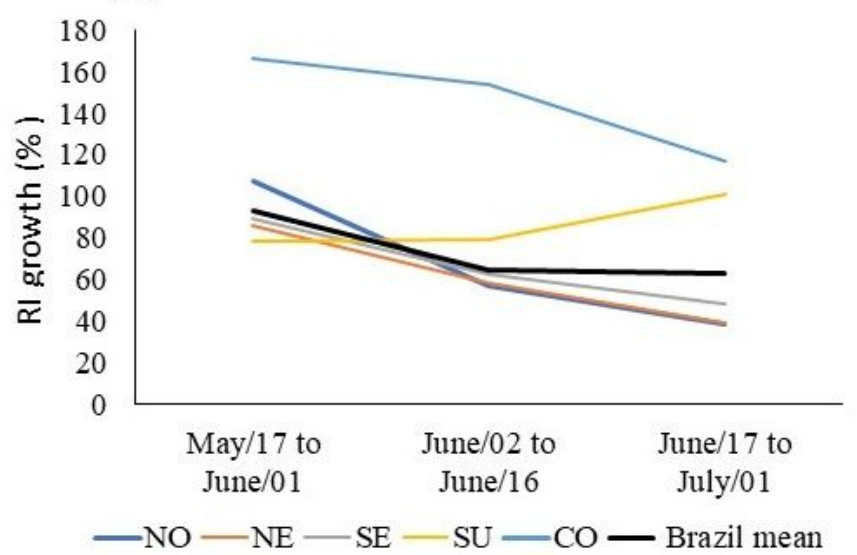

(B)

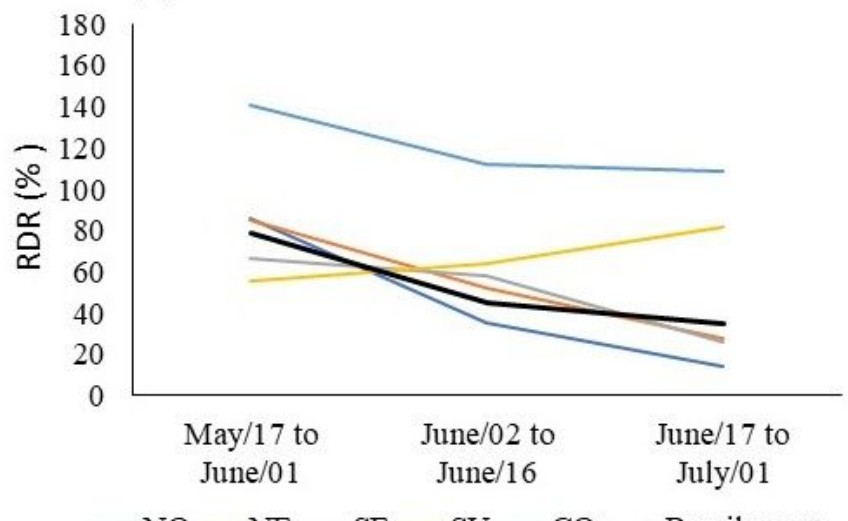

Figure 2

Acceleration rate of relative incidence (RI) growth and relative death rate (RDR) between May 17 and June 1, 2020, between June 2 and June 16, 2020, and between June 17 and July 01, 2020in north (N), northeast (NE), southeast (SE), south (S), and central weast (CW) region.
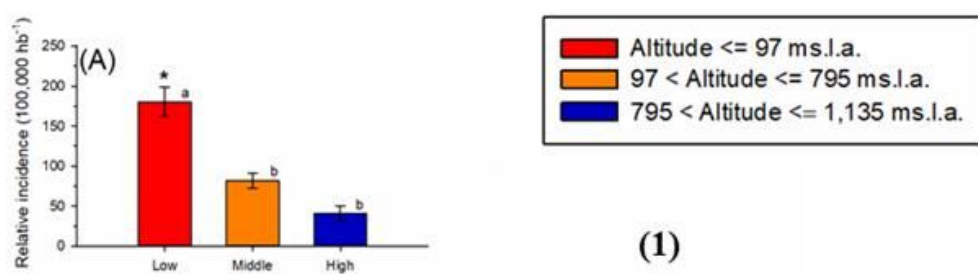

(1)
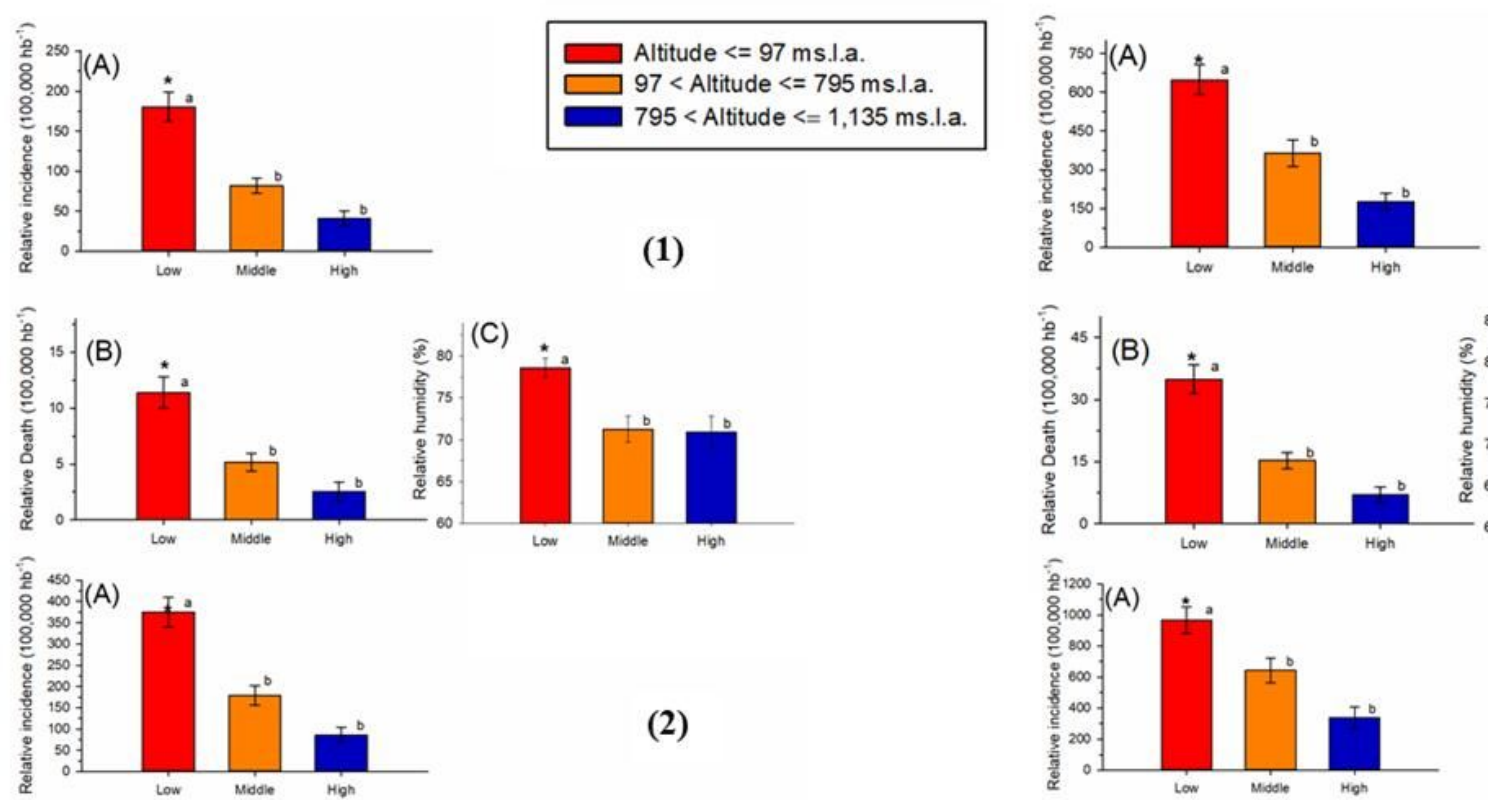

(2)

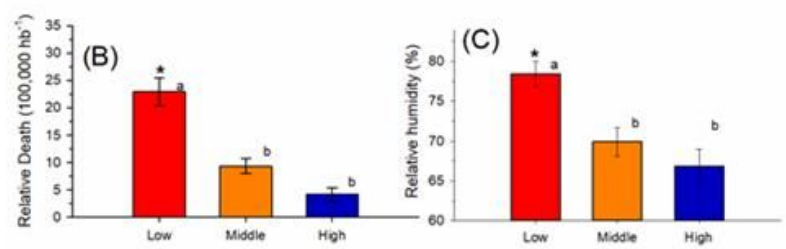

(3)
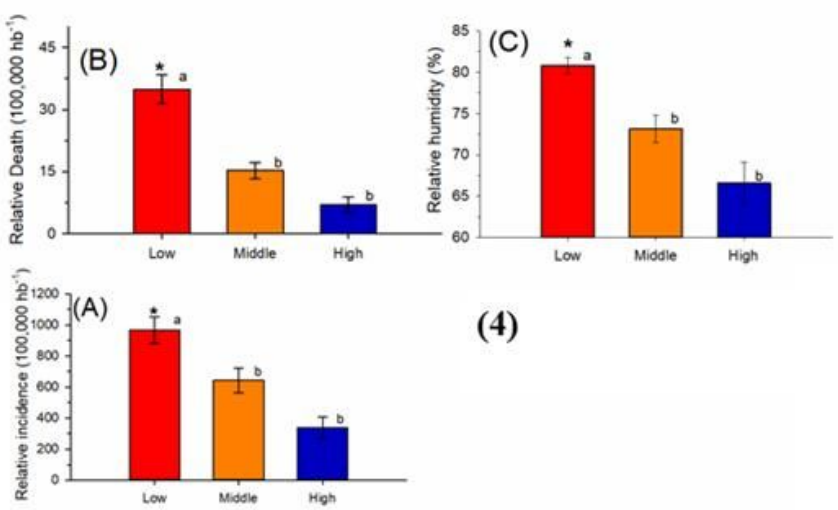

(4)

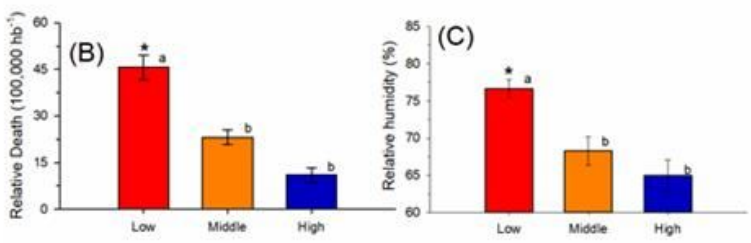

\section{Figure 3}

Acceleration rate of relative incidence (RI) growth and relative death rate (RDR) between May 17 and June 1, 2020, between June 2 and June 16, 2020, and between June 17 and July 01, 2020in north (N), northeast (NE), southeast (SE), south (S), and central weast (CW) region. 